\title{
Equivalent 2-D nonsingular Roesser models for discrete linear repetitive processes
}

\author{
Mohamed S. Boudellioua \\ Department of Mathematics and Statistics \\ Sultan Qaboos University \\ Muscat, Oman \\ Krzysztof Galkowski \\ Institute of Control and Computation Engineering \\ University of Zielona Gora \\ Zielona Gora, Szafrana 2, Poland \\ Corresponding author: k.galkowski@issi.uz.zgora.pl \\ Eric Rogers
Department of Electronics and Computer Science
University of Southampton, Southampton SO17 1BJ, UK
}

\begin{abstract}
The elementary operations algorithm is used to establish that a system matrix describing a discrete linear repetitive process can be transformed to that of a 2-D nonsingular Roesser model where all the input-output properties are preserved. Moreover, the connection between these system matrices is shown to be input-output equivalence. The exact forms of the resulting system matrix and the transformation involved are established. Some areas for possible future use/application of the developed results are also briefly discussed.

Keywords Linear repetitive processes, 2-D discrete systems, System matrix, 2-D non-singular Roesser form, Input-output equivalence.
\end{abstract}

\section{Introduction}

A 2-D system propagates information in two independent directions, which can be either time/space or space/space variables. Representations of the dynamics of 2-D systems include [1], the Roesser state-space model [16] and the Fornasini Marchesini state-space model [4]. The common feature of these models is that 
the describing equations are differential, difference or differential-difference in two independent variables. In the case of linear dynamics, the Laplace and/or $z$ transforms can be applied to obtain rational transfer function/matrix system representations for the dynamics. In this case, the resulting transfer-function (or entries in the transfer-function) matrix are functions of two complex variables. This, in turn, leads to complications in developing a systems matrix based theory for them as coprimeness, the key underpinning mathematics in such an approach, see, e.g., [18] for standard, also known as 1-D in some of the multidimensional systems theory literature, case, is no longer a single concept.

As in the 1-D case, conversion of a state-space model of the dynamics to a transfer-function matrix description and vice versa is a critical issue for 2-D systems described by the Roesser and Fornasini-Marchesini models, where again the situation is more complex than the 1-D case, especially in constructing a state-space model from a transfer-function matrix. One alternative approach is to apply the EOA algorithm [5] based on the application of elementary operations to multivariate functions that define the transfer-function entries. More recent work on this approach includes, e.g., [19] and the references therein.

Linear repetitive processes, see e.g., [17], are a particular case of 2-D systems. Such systems have attracted a great deal of interest in control theory and its applications and possess some distinct features relative to other classes of 2-D linear systems. These processes are the subject of this paper and the relevant background on them is given in the next section.

A very important task in 2-D systems theory is the ability to transform a given representation to an equivalent that can simplify subsequent analysis, e.g., the existence and design of stabilizing control laws. Previous work $[6,7]$ has transformed the dynamics of discrete linear repetitive processes to an equivalent Roesser or Fornasini-Marchesini 2-D state-space model and then used these models to characterize systems theoretic properties such as local reachability/controllability. However, the final conditions obtained in this previous work have a complicated form and are difficult to apply. The aim of this paper is to develop new 2-D equivalent models that may be easier to apply.

Previous work [2] developed a direct method for converting a polynomial matrix description of the dynamics of linear repetitive processes to those an equivalent 2-D singular state-space model using the EOA. For 2-D linear systems, only a singular state-space model can exist when a bi-variate system characteristic polynomial of degree $m, n$ in the complex variables $z_{1}, z_{2}$, respectively, has the zero coefficient associated with the term $z_{1}^{m} z_{2}^{n}$. However, the method of [2] produces a singular Roesser model in all cases, i.e., even when there exists a nonsingular model, which may be the source of many difficulties in further analysis.

In this paper, the results in [2] are extended to obtain a nonsingular Roesser model. The structure of the resulting system matrix is given and the transformation linking it to the original system matrix is established. It is shown that this transformation is input-output equivalence, which has been studied in the 2-D systems case by many authors, e.g., [12], [10] and [15, 14].

The paper is organized as follows: Sections 2 and 3, respectively, give the re- 
quired background on discrete linear repetitive processes, including a discussion of the differences with the Roesser model, and system equivalence. Sections 4 and 5, respectively, gives the system matrices for discrete linear repetitive processes and an overview of the EOA algorithm. Sections 6 and 7 give the main new results of this paper where it is shown how to transform a description of the dynamics of a discrete linear repetitive process to the nonsingular Roesser form by two methods, variables inversion (Section 6) and through use of the bilinear transform (Section 7), respectively, where in both cases the input-output equivalence of the resulting representations is established. The paper concludes in Section 8 with a discussion of the significance of these new results together with some areas for possible future research.

Except where explicitly stated the identity and null matrices with compatible dimensions are denoted in this paper by $I$ and 0 respectively.

\section{Discrete Linear Repetitive Processes as 2-D Systems}

Repetitive processes make a series of sweeps, or passes, through dynamics defined over a finite duration known as the finite pass length. Once each pass is complete, the process resets to the starting location and the next pass can begin, either immediately after the resetting is complete or after a further period of time has elapsed. On each pass the output produced is termed the pass profile and the unique characteristic of the these processes is that the previous pass profile acts as a forcing function on the next pass and hence contributes to its dynamics. The result can be oscillations that increase in amplitude from pass-to-pass and cannot be removed by application of standard control action.

These processes have their origins in the modeling for control of long-wall coal cutting operations where the original references are cited in [17]. Also a stability theory for linear examples has been developed based on an abstract model of the dynamics in a Banach space setting that includes all examples with linear dynamics and a constant pass length as special cases. This theory is of the bounded-input bounded-output form, i.e., a bounded initial pass profiles is required to produce a bounded sequence of pass profiles, either over the finite and fixed pass length or, in stronger form, for all possible pass lengths, with boundedness defined in terms of the norm on the function space in which the pass profile is assumed to lie.

In a number of important sub-classes, including the case considered in this paper, the stability theory has been refined to computable tests and algorithms for control law design. This, in turn, has led to the design of iterative learning control laws with supporting experimental verification of iterative learning control laws, see, e.g., $[8,9]$. Moreover, an extension to robust control with experimental verification has also been reported, see. e.g., [13].

The discrete linear repetitive processes considered in this paper are de- 
scribed [17] by the state-space model:

$$
\begin{aligned}
x_{k+1}(p+1) & =A x_{k+1}(p)+B_{0} y_{k}(p)+B u_{k+1}(p), \\
y_{k+1}(p) & =C x_{k+1}(p)+D_{0} y_{k}(p)+D u_{k+1}(p)
\end{aligned}
$$

where the indeterminate $p$ denotes the sample number along the pass $0 \leq p \leq$ $\alpha-1$, where $\alpha$ is the fixed number of samples along the pass, the indeterminate $k \geq 0$ denotes the pass number, $x_{k}(p) \in \mathbb{R}^{n}$ is the state vector, $y_{k}(p) \in \mathbb{R}^{m}$ is the pass profile vector, which also serves as a system output, and $u_{k}(p) \in \mathbb{R}^{l}$ is the input vector. To complete the process description, it is necessary to specify the boundary conditions, i.e., the state initial vector on each pass and the initial pass profile (i.e. on the 0th pass). For the purposes of this paper, it is assumed that the state initial vector at the start of each new pass is of the form $x_{k+1}(0)=d_{k+1}, k \geq 0$ and $y_{0}(p)=f(p), 0 \leq p \leq \alpha-1$ where the $n \times 1$ vector $d_{k+1}$ has known constant entries and those in the $m \times 1$ vector $f(p)$ are known functions of $p$. Without loss of generality, this paper will consider the case of zero boundary conditions.

The Roesser state-space model [16] for 2-D discrete recursive linear systems over the complete upper right quadrant of the 2-D plane has the following form where $x^{h}(i, j) \in \mathbb{R}^{n}$ and $x^{v}(i, j) \in \mathbb{R}^{m}$ are the so-called horizontal and vertical state vectors

$$
\begin{aligned}
{\left[\begin{array}{l}
x^{h}(i+1, j) \\
x^{v}(i, j+1)
\end{array}\right] } & =\left[\begin{array}{ll}
A_{11} & A_{12} \\
A_{21} & A_{22}
\end{array}\right]\left[\begin{array}{l}
x^{h}(i, j) \\
x^{v}(i, j)
\end{array}\right]+\left[\begin{array}{l}
B_{1} \\
B_{2}
\end{array}\right] u(i, j), \\
y(i, j) & =\left[\begin{array}{ll}
C_{1} & C_{2}
\end{array}\right]\left[\begin{array}{l}
x^{h}(i, j) \\
x^{v}(i, j)
\end{array}\right]+\mathbb{D} u(i, j)
\end{aligned}
$$

and $y(i, j) \in \mathbb{R}^{q}$ is the output vector and $u(i, j) \in \mathbb{R}^{l}$ the input vector. Again, zero boundary conditions are assumed, i.e., for $x^{h}(i, 0), i \geq 0$ and $x^{v}(0, j), j \geq 0$.

The Roesser and discrete linear repetitive process models (2) and (1), respectively, have similarities in terms of their structures but there are essential differences. The first is that repetitive processes are defined over a finite strip, or subset, of the upper right quadrant of the 2-D plane. In a repetitive process the 2-D systems structure arises from the influence of the previous pass profile on the current pass state and pass profile vectors, i.e., from the terms $B_{0} y_{k}(p)$ and $D_{0} y_{k}(p)$ in $(1)$ respectively. The differences between these two models are highlighted in Figure 1.

Systems theoretic properties relevant to applications for repetitive processes are known that have no Roesser model equivalents and the converse statement is also true. In Roesser model analysis, point-to-point controllability can be defined as: Given arbitrarily chosen initial and terminal state vectors is it possible to find a finite duration control signal that drives the system from the specified initial to terminal state vectors? This property is of relatively little interest in repetitive processes [6] and a more relevant property is pass profile controllability, see also [6]. Pass profile controllability requires the existence of an admissible control input sequence such that a specified pass profile can be achieved from the initial pass profile in finite number of passes. 

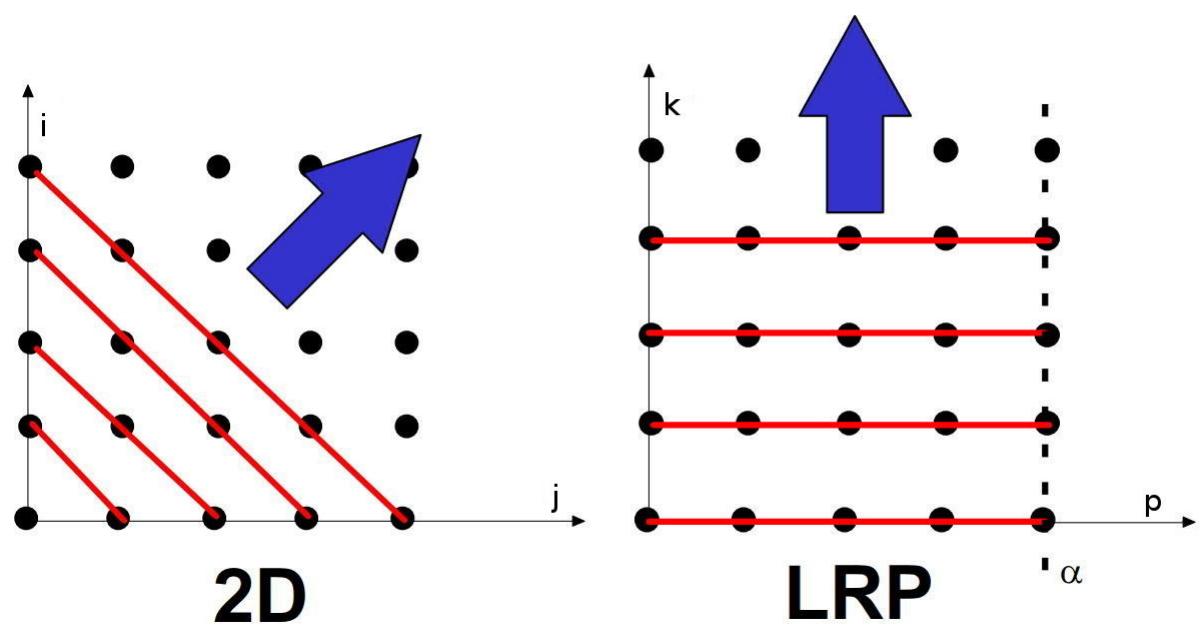

Figure 1: Evolution of the dynamics of a discrete linear repetitive process and the Roesser model

Conditions for pass profile controllability of processes described by (1) can be obtained by introducing super-vectors $X_{k}, Y_{k}$ and $U_{k}$, respectively. These vectors have entries defined by assembling the corresponding vector at each of the finite number of samples along the trial. Consider for simplicity the single-input single-output case then $Y_{k}=\left[\begin{array}{llll}y_{k}(0) & y_{k}(1) & \ldots & y_{k}(\alpha-1)\end{array}\right]^{T}$ and likewise for $X_{k}$ and $U_{k}$. As a physical example, various forms of metal rolling can be modeled as a repetitive process and the property of pass profile controllability can be formulated as reducing the thickness of the metal bar to a specified profile after a finite number of passes through the rollers.

Pass profile controllability has no Roesser model equivalent. Moreover, if this property is present then it guarantees the existence of a control law such that the controlled result has the required pass profile on the required pass. Also a weaker form of this property can be defined where the requirement of a specified pass number can be replaced by the requirement that the required pass profile is produced on some pass during the evolution of the dynamics.

\section{System Equivalence}

The concept of a polynomial system matrix was first introduced in, e.g., [18] for 1-D linear systems. The natural generalization to 2-D linear systems is the polynomial system description:

$$
\begin{aligned}
T\left(z_{1}, z_{2}\right) x & =U\left(z_{1}, z_{2}\right) u \\
y & =V\left(z_{1}, z_{2}\right) x+W\left(z_{1}, z_{2}\right) u
\end{aligned}
$$


where $x \in \mathbb{R}^{n}$ is the state vector, $u \in \mathbb{R}^{l}$ is the input vector and $y \in \mathbb{R}^{m}$ is the output vector, $T, U, V$ and $W$ are polynomial matrices with elements in $\mathbb{R}\left[z_{1}, z_{2}\right]$ of dimensions $n \times n, n \times l, m \times n$ and $m \times l$ respectively. The operators $z_{1}$ and $z_{2}$ may have various meanings depending on the type of system. For example, in delay-differential systems $z_{1}$ represents a differential operator and $z_{2}$ a delayoperator. For 2-D discrete systems, $z_{1}$ and $z_{2}$ represent horizontal and vertical shift operators, respectively and is the only case considered in this paper.

The system (3) gives rise to the system matrix in the general form:

$$
P\left(z_{1}, z_{2}\right)=\left[\begin{array}{rr}
T\left(z_{1}, z_{2}\right) & U\left(z_{1}, z_{2}\right) \\
-V\left(z_{1}, z_{2}\right) & W\left(z_{1}, z_{2}\right)
\end{array}\right]
$$

where

$$
P\left(z_{1}, z_{2}\right)\left[\begin{array}{r}
x \\
-u
\end{array}\right]=\left[\begin{array}{r}
0 \\
-y
\end{array}\right] .
$$

If $T\left(z_{1}, z_{2}\right)$ is invertible, the system matrix in (4) is said to be regular. The transfer-function matrix corresponding to the system matrix in (4) is given by:

$$
G\left(z_{1}, z_{2}\right)=V\left(z_{1}, z_{2}\right) T^{-1}\left(z_{1}, z_{2}\right) U\left(z_{1}, z_{2}\right)+W\left(z_{1}, z_{2}\right)
$$

One of the most fundamental requirements for the equivalence of two system matrices is the preservation of the input-output (I/O) behavior. Invariance of the I/O behavior is ensured by the equality of the transfer-function matrices.

Definition 1 [11] Let $\mathbb{T}(m, n)$ denote the class of $(r+m) \times(r+n)$ rational matrices where $r>\min (m, n)$. The subset $\mathbb{P}(m, n)$ of $\mathbb{T}(m, n)$ obtained by requiring $r>0$ represents the 2-D rational system matrices. Two system matrices $P_{1}\left(z_{1}, z_{2}\right)$ and $P_{2}\left(z_{1}, z_{2}\right) \in \mathbb{P}(m, n)$, are said to be $I / O$ equivalent if they have the same transfer-function matrix, i.e.,

$$
G_{1}\left(z_{1}, z_{2}\right)=G_{2}\left(z_{1}, z_{2}\right)
$$

The following is a characterization of $\mathrm{I} / \mathrm{O}$ equivalence.

Lemma 1 [11] Two system matrices $P_{1}\left(z_{1}, z_{2}\right)$ and $P_{2}\left(z_{1}, z_{2}\right) \in \mathbb{P}(m, n)$, are $I / O$ equivalent if and only if there exist rational matrices $M\left(z_{1}, z_{2}\right), N\left(z_{1}, z_{2}\right)$, $X\left(z_{1}, z_{2}\right)$, and $Y\left(z_{1}, z_{2}\right)$ such that

$$
\begin{gathered}
{\left[\begin{array}{cc}
M\left(z_{1}, z_{2}\right) & 0 \\
X\left(z_{1}, z_{2}\right) & I
\end{array}\right]\left[\begin{array}{cc}
T_{2}\left(z_{1}, z_{2}\right) & U_{2}\left(z_{1}, z_{2}\right) \\
-V_{2}\left(z_{1}, z_{2}\right) & W_{2}\left(z_{1}, z_{2}\right)
\end{array}\right]=A\left(z_{1}, z_{2}\right)} \\
A\left(z_{1}, z_{2}\right)=\left[\begin{array}{cc}
T_{1}\left(z_{1}, z_{2}\right) & U_{1}\left(z_{1}, z_{2}\right) \\
-V_{1}\left(z_{1}, z_{2}\right) & W_{1}\left(z_{1}, z_{2}\right)
\end{array}\right]\left[\begin{array}{cc}
N\left(z_{1}, z_{2}\right) & Y\left(z_{1}, z_{2}\right) \\
0 & I
\end{array}\right] .
\end{gathered}
$$




\section{System Matrices for Linear Repetitive Pro- cesses}

In this section discrete linear repetitive processes described by (1) are considered. Introduce the forward shift operators $z_{1}$ in the pass-to-pass direction and $z_{2}$ in the along the pass direction, i.e.,

$$
z_{1} s_{k}(p)=s_{k+1}(p), \quad z_{2} s_{k}(p)=s_{k}(p+1)
$$

respectively, where the signal $s_{k}(p)$ represents $x_{k}(p)$ or $y_{k}(p)$ as appropriate. The process dynamics with zero boundary conditions can now be written in polynomial matrix form as:

$$
P_{R P}\left(z_{1}, z_{2}\right)\left[\begin{array}{r}
x_{k}(p) \\
y_{k}(p) \\
\hline-u_{k}(p)
\end{array}\right]=\left[\begin{array}{c}
0 \\
0 \\
\hline-y_{k}(p)
\end{array}\right]
$$

where

$$
P_{R P}\left(z_{1}, z_{2}\right)=\left[\begin{array}{cc|c}
z_{1} z_{2} I-z_{1} A & -B_{0} & z_{1} B \\
-z_{1} C & z_{1} I-D_{0} & z_{1} D \\
\hline 0 & -I_{m} & 0
\end{array}\right]
$$

is the system matrix associated with (1). Alternatively the dynamics of (1) can be represented in transfer-function matrix form as:

$$
Y\left(z_{1}, z_{2}\right)=G_{R P}\left(z_{1}, z_{2}\right) U\left(z_{1}, z_{2}\right)
$$

where

$$
G_{R P}\left(z_{1}, z_{2}\right)=\left[\begin{array}{ll}
0 & I
\end{array}\right]\left[\begin{array}{cc}
z_{1} z_{2} I-z_{1} A & -B_{0} \\
-z_{1} C & z_{1} I-D_{0}
\end{array}\right]^{-1}\left[\begin{array}{c}
z_{1} B \\
z_{1} D
\end{array}\right] .
$$

Example 1 Consider the discrete repetitive process with state-space model matrices:

$$
\begin{gathered}
A=\left[\begin{array}{cc}
1 & -1 \\
2 & 0
\end{array}\right], \quad B_{0}=\left[\begin{array}{cc}
0 & 1 \\
2 & 1
\end{array}\right], \quad B=\left[\begin{array}{cc}
1 & 0 \\
1 & 1
\end{array}\right], \\
C=\left[\begin{array}{ll}
0 & 0 \\
0 & 1
\end{array}\right], \quad D=\left[\begin{array}{cc}
1 & 0 \\
0 & -1
\end{array}\right], \quad D_{0}=\left[\begin{array}{cc}
-1 & 0 \\
0 & 0
\end{array}\right] .
\end{gathered}
$$

The polynomial system matrix corresponding to (15) is:

$$
P_{R P}\left(z_{1}, z_{2}\right)=\left[\begin{array}{cccc|cc}
z_{1} z_{2}-z_{1} & z_{1} & 0 & -1 & z_{1} & 0 \\
-2 z_{1} & z_{1} z_{2} & -2 & -1 & z_{1} & z_{1} \\
0 & 0 & z_{1}+1 & 0 & z_{1} & 0 \\
0 & -z_{1} & 0 & z_{1} & 0 & -z_{1} \\
\hline 0 & 0 & -1 & 0 & 0 & 0 \\
0 & 0 & 0 & -1 & 0 & 0
\end{array}\right]
$$

and the 2-D transfer-function matrix is:

$$
G_{R P}\left(z_{1}, z_{2}\right)=\left[\begin{array}{c}
\frac{z_{1}}{z_{1}+1} \\
\frac{z_{1}\left(z_{1} z_{2}+z_{1}+3 z_{2}-1\right)}{\left(z_{1}+1\right)\left(z_{1} z_{2}{ }^{2}-z_{1} z_{2}+2 z_{1}-z_{2}-1\right)}-\frac{z_{1}\left(z_{2}^{2}-2 z_{2}+3\right)}{z_{1} z_{2}^{2}-z_{1} z_{2}+2 z_{1}-z_{2}-1}
\end{array}\right] .
$$




\section{$5 \quad$ EOA Basics}

The EOA, see [5] is a method for obtaining a state-space model description of a system from it 2-D transfer-function matrix. Next the EOA is detailed for square transfer-function matrices, which does not limit the generality of the results (the rectangular case is detailed in [5], which also describes the extension to $n$-D, $n \geq 3$, systems).

Rewrite a given 2-D transfer function matrix, say $F\left(z_{1}, z_{2}\right)$, in the form:

$$
F\left(z_{1}, z_{2}\right)=\left[\begin{array}{ccc}
\cdots & \cdots & \cdots \\
\vdots & \frac{b_{i j}\left(z_{1}, z_{2}\right)}{a_{i j}\left(z_{1}, z_{2}\right)} & \vdots \\
\cdots & \cdots & \ldots
\end{array}\right]=\left[\begin{array}{ccc}
\cdots & \cdots & \cdots \\
\vdots & \frac{B_{i j}\left(z_{1}, z_{2}\right)}{A_{j}\left(z_{1}, z_{2}\right)} & \vdots \\
\cdots & \cdots & \ldots
\end{array}\right]
$$

where $A_{j}\left(z_{1}, z_{2}\right)$ is the least common multiple of the denominators of all the entries in the $j$ th column of the transfer-function matrix, which is equivalent to the matrix fraction description:

$$
F\left(z_{1}, z_{2}\right)=N_{R}\left(z_{1}, z_{2}\right) D_{R}\left(z_{1}, z_{2}\right)^{-1}
$$

with a diagonal polynomial matrix $D_{R}\left(z_{1}, z_{2}\right)$. For example, the transfer-function given by (17) can be written as

$$
G_{R P}\left(z_{1}, z_{2}\right)=\left[\begin{array}{cc}
\frac{z_{1}\left(z_{1} z_{2}^{2}-z_{1} z_{2}+2 z_{1}-z_{2}-1\right)}{\left(z_{1}+1\right)\left(z_{1} z_{2}^{2}-z_{1} z_{2}+2 z_{1}-z_{2}-1\right)} & 0 \\
\frac{z_{1}\left(z_{1} z_{2}+z_{1}+3 z_{2}-1\right)}{\left(z_{1}+1\right)\left(z_{1} z_{2}^{2}-z_{1} z_{2}+2 z_{1}-z_{2}-1\right)} & -\frac{z_{1}\left(z_{2}^{2}-2 z_{2}+3\right)}{z_{1} z_{2}^{2}-z_{1} z_{2}+2 z_{1}-z_{2}-1}
\end{array}\right],
$$

and hence $A_{1}\left(z_{1}, z_{2}\right)=\left(z_{1}+1\right)\left(z_{1} z_{2}^{2}-z_{1} z_{2}+2 z_{1}-z_{2}-1\right)$ and $A_{2}\left(z_{1}, z_{2}\right)=$ $z_{1} z_{2}^{2}-z_{1} z_{2}+2 z_{1}-z_{2}-1$. It is also possible to use a dual approach where the least common multiple of the denominators of all the entries in the $j$ th row of the transfer-function matrix is used.

The next step is to represent a 2 -D transfer-function matrix in the form of an associated polynomial matrix where auxiliary variables are added. In [5] a number of alternative approaches were developed but this section only considers one of them which is suitable for application to discrete linear repetitive processes. In particular, construct from (18) the following polynomial matrix

$$
A_{F}\left(z_{1}, z_{2}, z\right)=\left[\begin{array}{cccc}
z A_{1}\left(z_{1}, z_{2}\right)-B_{11}\left(z_{1}, z_{2}\right) & \cdots & \cdots & -B_{1 m}\left(z_{1}, z_{2}\right) \\
-B_{21}\left(z_{1}, z_{2}\right) & \cdots & \cdots & -B_{2 m}\left(z_{1}, z_{2}\right) \\
\vdots & \vdots & \ddots & \vdots \\
-B_{m 1}\left(z_{1}, z_{2}\right) & \cdots & \cdots & z A_{m}\left(z_{1}, z_{2}\right)-B_{m m}\left(z_{1}, z_{2}\right)
\end{array}\right]
$$

where the auxiliary variable $z$ has been inserted to split the denominator and numerator polynomials of each entry and is removed when the procedure is complete.

The idea of the EOA is to apply to $A_{F}\left(z_{1}, z_{2}, z\right)$ of (21) a sequence of size augmentation operations followed by elementary row and column operations to lower the polynomial degrees and split different variables to obtain a matrix 
whose entries are first order polynomials in a single variable. Further application of appropriate row and column permutations produces the required system matrix associated with the Roesser state-space model. Specifically, the following matrix operations are introduced:

$$
Y=\operatorname{Augment}(X)=\left[\begin{array}{ll}
1 & 0 \\
0 & X
\end{array}\right],
$$

- $Y=\operatorname{addrow}(X, i, j, \phi)$ - multiply the $i$ th row of $X$ by $\phi$ and add the result to the $j$ th row of $X$,

- $Y=\operatorname{addcol}(X, i, j, \phi)$ - multiply the $i$ th column of $X$ by $\phi$ and add the result to the $j$ th column of $X$,

- $Y=\operatorname{mulrow}(X, i, \phi), Y=\operatorname{mulcol}(X, i, \phi)-$ multiply the $i$ th row $/$ column of $X$ respectively by $\phi$.

Finally, a series of row and column permutations must be applied to obtain appropriate variable ordering.

Direct application of this method to linear repetitive processes will lead, in most cases, to a singular Roesser model, see [2]. The next section shows that this deficiency can be avoided by applying variable transformations, such as inversion of variables or a generalized bilinear transform.

\section{Transformation of a Discrete Linear Repeti- tive Process to the Nonsingular Roesser Form Using Variable Inversion}

In [2] an equivalence between discrete linear repetitive processes and a singular 2-D Roesser model was established. Moreover, it was shown in [5] that, in some cases, the singular 2-D Roesser model can be transformed to one that is nonsingular by using the inverses of the variables $z_{1}$ and $z_{2}$, i.e., the substitutions $z_{1}=\widehat{z}_{1}^{-1}$ and $z_{2}=\widehat{z}_{2}^{-1}$. However, the inverted variables $\widehat{z}_{i}, \quad i=1,2$ correspond to a reversed time shift and the resulting system would be a'causal and hence not physically realizable.

To overcome this difficulty, the route in this paper is to first invert variables in the transfer-function matrix considered, i.e., make the substitutions $z_{1}=\widehat{z}_{1}^{-1}$ and $z_{2}=\widehat{z}_{2}^{-1}$, then obtain the auxiliary singular Roesser model using the results in [2] and [5] and then return to the original variables to obtain a nonsingular Roesser model. The result is a state-space model that can be written in the form

$$
\begin{gathered}
E^{\prime}\left[\begin{array}{c}
\widehat{x}_{1}(i+1, j) \\
\widehat{x}_{2}(i, j+1)
\end{array}\right]=\left[\begin{array}{ll}
H_{11} & H_{12} \\
H_{21} & H_{22}
\end{array}\right]\left[\begin{array}{l}
\widehat{x}_{1}(i, j) \\
\widehat{x}_{2}(i, j)
\end{array}\right]+\left[\begin{array}{c}
H_{13} \\
H_{23}
\end{array}\right] \widehat{u}(i, j), \\
\widehat{y}(i, j)=\left[\begin{array}{ll}
H_{31} & H_{32}
\end{array}\right]\left[\begin{array}{l}
\widehat{x}_{1}(i, j) \\
\widehat{x}_{2}(i, j)
\end{array}\right]+H_{33} \widehat{u}(i, j)
\end{gathered}
$$


where $E^{\prime}=0 \oplus I$ and $\oplus$ denotes the direct sum of two matrices. Here, although the variables have been inverted, the model is written in the commonly used way to relate it to the transfer-function matrix obtained using $\widehat{z}_{i}, i=1,2$.

Introduce the following matrices

$$
\begin{aligned}
\widehat{H}_{11} & =\left[\begin{array}{ll}
H_{11} & H_{12} \\
H_{21} & H_{22}
\end{array}\right], \widehat{H}_{12}=\left[\begin{array}{l}
H_{13} \\
H_{23}
\end{array}\right], \\
\widehat{H}_{21} & =\left[\begin{array}{ll}
H_{31} & H_{32}
\end{array}\right], \widehat{H}_{22}=H_{33}
\end{aligned}
$$

and introduce the substitutions $\widehat{z}_{1}^{-1}=z_{1}, \widehat{z}_{2}^{-1}=z_{2}$ to obtain a nonsingular Roesser model

$$
\begin{array}{r}
{\left[\begin{array}{ll}
A_{11} & A_{12} \\
A_{21} & A_{22}
\end{array}\right]=\widehat{H}_{11}^{-1} E^{\prime}, \quad\left[\begin{array}{l}
B_{1} \\
B_{2}
\end{array}\right]=-\widehat{H}_{11}^{-1} \widehat{H}_{12},} \\
{\left[\begin{array}{ll}
C_{1} & C_{2}
\end{array}\right]=\widehat{H}_{21} \widehat{H}_{11}^{-1} E^{\prime}, \mathbb{D}=\widehat{H}_{22}-\widehat{H}_{21} \widehat{H}_{11}^{-1} E^{\prime} \widehat{H}_{12}}
\end{array}
$$

provided that $\widehat{H}_{11}$ is nonsingular. The resulting system matrix in the nonsingular Roesser form corresponding to a discrete linear repetitive process (12) is given by

$$
P_{R O}=\left[\begin{array}{cc|c}
z_{1} I_{N}-A_{11} & -A_{12} & B_{1} \\
-A_{21} & z_{2} I_{M}-A_{22} & B_{2} \\
\hline-C_{1} & -C_{2} & \mathbb{D}
\end{array}\right]
$$

where $N \geq n, M \geq m$.

Theorem 1 Let $P_{R P}\left(z_{1}, z_{2}\right)$ be the $[(n+m)+m] \times[(n+m)+p]$ polynomial system matrix given by (12). Then $P_{R P}\left(z_{1}, z_{2}\right)$ is $I / O$ equivalent to a nonsingular Roesser model system matrix of the form (26), provided that this representation exists, i.e.,

$$
S_{1}\left(z_{1}, z_{2}\right) P_{R P}\left(z_{1}, z_{2}\right)=P_{R O}\left(z_{1}, z_{2}\right) S_{2}\left(z_{1}, z_{2}\right)
$$

where

$$
S_{1}\left(z_{1}, z_{2}\right)=\left[\begin{array}{cc}
M\left(z_{1}, z_{2}\right) & 0 \\
X\left(z_{1}, z_{2}\right) & I
\end{array}\right], \quad S_{2}\left(z_{1}, z_{2}\right)=\left[\begin{array}{cc}
M\left(z_{1}, z_{2}\right) & Y\left(z_{1}, z_{2}\right) \\
0 & I
\end{array}\right]
$$

are matrices with elements in $\mathbb{R}\left(z_{1}, z_{2}\right)$.

Proof 1 As shown in [2], a polynomial system matrix associated with a discrete linear repetitive process is zero coprime system equivalent and hence I/O equivalent to a polynomial system matrix associated with a singular Roesser model described by (23). Conversely, the double inversion of variables preserves a transfer-function matrix. Finally, (27) and (28) follow from Lemma 1.

Example 2 Consider again the system of Example 1. First apply the substitutions $z_{1}=\widehat{z}_{1}^{-1}$ and $z_{2}=\widehat{z}_{2}^{-1}$ to the transfer-function matrix $G_{R P}\left(z_{1}, z_{2}\right)$ to 
obtain

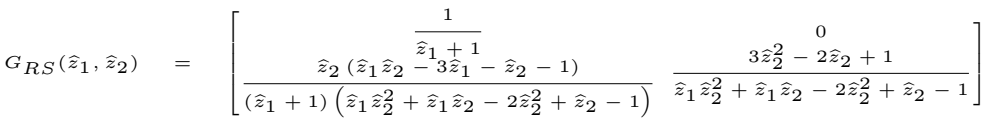

$$
\begin{aligned}
& =\left[\begin{array}{cc}
\frac{\widehat{z}_{1} \widehat{z}_{2}^{2}+\widehat{z}_{1} \widehat{z}_{2}-2 \widehat{z}_{2}^{2}+\widehat{z}_{2}-1}{\left(\widehat{z}_{1}+1\right)\left(\widehat{z}_{1} \hat{z}_{2}^{2}+\widehat{z}_{1} \widehat{z}_{2}-2 \widehat{z}_{2}^{2}+\widehat{z}_{2}-1\right)} & 0 \\
\frac{\widehat{z}_{2}\left(\widehat{z}_{1} \widehat{z}_{2}-3 \widehat{z}_{1}-\widehat{z}_{2}-1\right)}{\left(\widehat{z}_{1}+1\right)\left(\widehat{z}_{1} \hat{z}_{2}^{2}+\widehat{z}_{1} \widehat{z}_{2}-2 \widehat{z}_{2}^{2}+\widehat{z}_{2}-1\right)} & \frac{0}{\widehat{z}_{1} \widehat{z}_{2}^{2}+\widehat{z}_{1} \widehat{z}_{2}-2 \widehat{z}_{2}^{2}+\widehat{z}_{2}-1}
\end{array}\right] .
\end{aligned}
$$

Using, for example the linalg Maple package, the following steps of the EOA can be performed, see [5]. First construct the 3-D polynomial matrix $\mathrm{H}_{0}$ (see (21))

$$
\begin{aligned}
& H_{0}=A_{F}\left(\widehat{z}_{1}, \widehat{z}_{2}, z\right) \\
& =\left[\begin{array}{c}
z\left(\widehat{z}_{1}+1\right)\left(\widehat{z}_{1} \widehat{z}_{2}^{2}-2 \widehat{z}_{2}^{2}+\widehat{z}_{1} \widehat{z}_{2}+\widehat{z}_{2}-1\right)-\left(\widehat{z}_{1} \widehat{z}_{2}^{2}-2 \widehat{z}_{2}^{2}+\widehat{z}_{1} \widehat{z}_{2}+\widehat{z}_{2}-1\right) \\
-\widehat{z}_{2}\left(\widehat{z}_{1} \widehat{z}_{2}-3 \widehat{z}_{1}-\widehat{z}_{2}-1\right)
\end{array}\right. \\
& 0 \\
& \left.z\left(\widehat{z}_{1} \widehat{z}_{2}^{2}-2 \widehat{z}_{2}^{2}+\widehat{z}_{1} \widehat{z}_{2}+\widehat{z}_{2}-1\right)-\left(3 \widehat{z}_{2}^{2}-2 \widehat{z}_{2}+1\right)\right]
\end{aligned}
$$

Next, perform the following sequence of operations, as defined in (22) and beneath,

$$
\begin{gathered}
H_{1}=\operatorname{Augment}\left(H_{0}\right), \quad H_{1}^{1}=\operatorname{addrow}\left(H_{1}, 1,2, z\right), \\
H_{1}^{2}=\operatorname{addcol}\left(H_{1}^{1}, 1,2,-\left(\widehat{z}_{1}+1\right)\left(\widehat{z}_{1} \widehat{z}_{2}^{2}-2 \widehat{z}_{2}^{2}+\widehat{z}_{1} \widehat{z}_{2}+\widehat{z}_{2}-1\right)\right), \\
H_{2}=\operatorname{Augment}\left(H_{1}^{2}\right), \quad H_{2}^{1}=\operatorname{addrow}\left(H_{2}, 1,4, z\right), \\
H_{3}=\operatorname{Augment}\left(H_{2}^{1}\right), H_{3}^{1}=\operatorname{addcol}\left(H_{3}, 1,4,\left(\widehat{z}_{1} \widehat{z}_{2}^{2}-2 \widehat{z}_{2}^{2}+\widehat{z}_{1} \widehat{z}_{2}+\widehat{z}_{2}-1\right)\right), \\
H_{3}^{2}=\operatorname{addrow}\left(H_{3}^{1}, 1,3,\left(\widehat{z}_{1}+1\right)\right), H_{3}^{3}=\operatorname{addrow}\left(H_{3}^{2}, 1,4,1\right), \\
H_{4}=\operatorname{Augment}\left(H_{3}^{3}\right), \quad H_{4}^{1}=\operatorname{addcol}\left(H_{4}, 1,5, \widehat{z}_{2}\right), \\
H_{5}=\operatorname{Augment}\left(H_{4}^{1}\right), H_{5}^{1}=\operatorname{addcol}\left(H_{5}, 1,2, \widehat{z}_{1}\right), \\
H_{5}^{2}=\operatorname{addrow}\left(H_{5}^{1}, 3,7,1\right), \quad H_{5}^{3}=\operatorname{addcol}\left(H_{5}^{2}, 1,2,-2\right), \\
H_{6}=\operatorname{Augment}\left(H_{5}^{3}\right), \quad H_{6}^{1}=\operatorname{addcol}\left(H_{6}, 1,8, \widehat{z}_{2}\right),
\end{gathered}
$$




$$
\begin{gathered}
H_{7}=\operatorname{Augment}\left(H_{6}^{1}\right), \quad H_{7}^{1}=\operatorname{addrow}\left(H_{7}, 1,6,\left(\widehat{z}_{1}-2\right)\right), H_{7}^{2}=\operatorname{addrow}\left(H_{7}^{1}, 1,9,3\right), \\
H_{8}=\operatorname{Augment}\left(H_{7}^{2}\right), H_{8}^{1}=\operatorname{addcol}\left(H_{8}, 1,5, \widehat{z}_{2}\right), \\
H_{9}=\operatorname{Augment}\left(H_{8}^{1}\right), \quad H_{9}^{1}=\operatorname{addrow}\left(H_{9}, 1,5, \widehat{z}_{1}\right), \\
H_{9}^{2}=\operatorname{addcol}\left(H_{9}^{1}, 1,6,-1\right), \quad H_{9}^{3}=\operatorname{mulrow}\left(H_{9}^{2}, 3,-1\right) .
\end{gathered}
$$

Finally, apply to the matrix $\mathrm{H}_{9}^{2}$ appropriate row and column permutations followed by block splitting to obtain the following singular Roesser state-space model matrices of the form given in (23)

$$
\begin{aligned}
& H_{11}=\left[\begin{array}{cccc}
0 & -1 & 0 & 0 \\
0 & 0 & 0 & 0 \\
0 & 0 & 2 & 0 \\
0 & 0 & 0 & -1
\end{array}\right], H_{12}=\left[\begin{array}{ccccc}
1 & 0 & 0 & 0 & 0 \\
2 & 0 & 0 & 0 & -1 \\
0 & -3 & -1 & 0 & 0 \\
0 & 0 & 0 & 0 & 0
\end{array}\right], \quad H_{13}=\left[\begin{array}{cc}
0 & 0 \\
0 & 0 \\
0 & -1 \\
-1 & 0
\end{array}\right] \text {, } \\
& H_{21}=\left[\begin{array}{cccc}
-1 & 0 & 0 & 0 \\
0 & 0 & 1 & 0 \\
0 & 0 & 0 & 0 \\
0 & 0 & 0 & 0 \\
0 & 0 & 0 & -1
\end{array}\right], H_{22}=\left[\begin{array}{ccccc}
0 & 0 & 0 & 0 & 0 \\
0 & -1 & 0 & 0 & 0 \\
0 & -1 & 0 & 0 & 0 \\
-1 & 0 & 0 & 0 & 0 \\
3 & 0 & 0 & 1 & -1
\end{array}\right], \quad H_{23}=\left[\begin{array}{ll}
0 & 0 \\
0 & 0 \\
0 & 0 \\
0 & 0 \\
0 & 0
\end{array}\right], \\
& H_{31}=\left[\begin{array}{cccc}
0 & 0 & 0 & -1 \\
1 & 0 & -3 & -1
\end{array}\right], \quad H_{32}=\left[\begin{array}{ccccc}
0 & 0 & 0 & 0 & 0 \\
10 & 5 & 1 & 1 & -4
\end{array}\right], \quad H_{33}=\left[\begin{array}{cc}
\frac{1}{3} & 0 \\
0 & 0
\end{array}\right]
\end{aligned}
$$

with $E^{\prime}=0 \oplus I_{8}$.

The matrices (25) of the nonsingular Roesser state-space model are

$$
\begin{aligned}
& A_{11}=\left[\begin{array}{cccc}
0 & 0 & 0 & 0 \\
0 & 0 & 0 & 0 \\
0 & 0 & 0 & 0 \\
0 & 0 & 0 & -1
\end{array}\right], A_{12}=\left[\begin{array}{ccccc}
-1 & 0 & 0 & 0 & 0 \\
0 & 0 & 0 & -1 & 0 \\
0 & 1 & -1 & 0 & 0 \\
0 & 0 & 0 & 0 & 0
\end{array}\right], B_{1}=\left[\begin{array}{cc}
0 & 0 \\
0 & 0 \\
0 & 0 \\
-1 & 0
\end{array}\right], \\
& A_{21}=\left[\begin{array}{cccc}
0 & 0 & 0 & 0 \\
0 & 0 & 0 & 0 \\
0 & 0 & -1 & 0 \\
0 & -1 & 0 & -1 \\
0 & -1 & 0 & 0
\end{array}\right], A_{22}=\left[\begin{array}{ccccc}
0 & 0 & 0 & -1 & 0 \\
0 & 0 & -1 & 0 & 0 \\
0 & 2 & 1 & 0 & 0 \\
0 & 0 & 0 & 1 & 1 \\
0 & 0 & 0 & -2 & 0
\end{array}\right], B_{2}=\left[\begin{array}{cc}
0 & 0 \\
0 & 0 \\
0 & -1 \\
-1 & 0 \\
0 & 0
\end{array}\right] \text {, } \\
& C_{1}=\left[\begin{array}{cccc}
0 & 0 & 0 & 1 \\
0 & 3 & -1 & 0
\end{array}\right], \quad C_{2}=\left[\begin{array}{ccccc}
0 & 0 & 0 & 0 & 0 \\
-1 & -1 & -1 & -1 & 1
\end{array}\right], \quad D=\left[\begin{array}{cc}
1 & 0 \\
0 & -1
\end{array}\right] \text {. }
\end{aligned}
$$

Finally, it is easily verified that the transfer-function matrix corresponding to this nonsingular Roesser state-space model is given by

$$
\begin{aligned}
G_{R O}\left(z_{1}, z_{2}\right) & =\left[\begin{array}{c}
\frac{z_{1}}{z_{1}+1} \\
\frac{z_{1}\left(z_{1} z_{2}+z_{1}+3 z_{2}-1\right)}{\left(z_{1}+1\right)\left(z_{1} z_{2}{ }^{2}-z_{1} z_{2}+2 z_{1}-z_{2}-1\right)}-\frac{z_{1}\left(z_{2}^{2}-2 z_{2}+3\right)}{z_{1} z_{2}^{2}-z_{1} z_{2}+2 z_{1}-z_{2}-1}
\end{array}\right] \\
& =G_{R P}\left(z_{1}, z_{2}\right) .
\end{aligned}
$$

The method developed above cannot be applied if the matrix $\widehat{H}_{11}$ is singular. In such cases, a generalized bilinear transform (see [5] can be used and its development is the subject of the next section. 


\section{Transformation of a Discrete Linear Repeti- tive Process to the Nonsingular Roesser Form Using a Generalized Bilinear Transform}

An alternative to variable inversion is to use the generalized bilinear transform [5], which can yield a nonsingular state-space model. However, this approach may also change the physical meaning of the system model, even in a more complex way than the inverse transform. Hence the route is to apply it twice; once to the initial transfer-function matrix and then to the singular system matrix obtained from applying the EOA procedure. On completing these steps, the original variables are recovered together with their mathematical and physical meanings.

The generalized multivariate bilinear transform is defined by the following pair of substitutions

$$
z_{i}=\frac{a_{i} \widetilde{z}_{i}+b_{i}}{c_{i} \widetilde{z}_{i}-a_{i}}, \quad \widetilde{z}_{i}=\frac{a_{i} z_{i}+b_{i}}{c_{i} z_{i}-a_{i}}, \quad a_{i}, b_{i}, c_{i} \in \mathbb{R}^{+}, \quad i=1,2 .
$$

and the EOA procedure is then applied to the transfer-function matrix of a linear repetitive process constructed using the transformed variables as given by (31) but excluding the auxiliary variable $z$. To obtain a singular Roesser model of the form (23) and (24), where instead of $\widehat{H}_{i j}, i, j=1,2$ the matrices $\widetilde{H}_{i j}$ are used to distinguish the two cases, i.e., the variable inversion and bilinear transform methods. It is routine to establish that applying the bilinear transform given in the second entry in (31) to the singular Roesser model produces a nonsingular model of the form (26) with the matrices

$$
\begin{gathered}
{\left[\begin{array}{ll}
A_{11} & A_{12} \\
A_{21} & A_{22}
\end{array}\right]=-\left(E^{\prime} \Lambda_{b}+\Lambda_{a} \widetilde{H}_{11}\right)\left(E^{\prime} \Lambda_{a}-\Lambda_{c} \widetilde{H}_{11}\right)^{-1}=\mathcal{A}} \\
{\left[\begin{array}{l}
B_{1} \\
B_{2}
\end{array}\right]=\left(\mathcal{A} \Lambda_{c}-\Lambda_{a}\right) \widetilde{H}_{12}} \\
{\left[\begin{array}{ll}
C_{1} & C_{2}
\end{array}\right]=\widetilde{H}_{21}\left(E^{\prime} \Lambda_{a}-\Lambda_{c} \widetilde{H}_{11}\right)^{-1}=\mathcal{C}} \\
\mathbb{D}=\widetilde{H}_{22}+\mathcal{C} \Lambda_{c} \widetilde{H}_{12}
\end{gathered}
$$

where

$$
\Lambda_{a}=\left[\begin{array}{cc}
a_{1} I_{N} & 0 \\
0 & a_{2} I_{M}
\end{array}\right], \Lambda_{b}=\left[\begin{array}{cc}
b_{1} I_{N} & 0 \\
0 & b_{2} I_{M}
\end{array}\right], \quad \Lambda_{a}=\left[\begin{array}{cc}
c_{1} I_{N} & 0 \\
0 & c_{2} I_{M}
\end{array}\right],
$$

provided that the matrix $E^{\prime} \Lambda_{a}-\Lambda_{c} \widetilde{H}_{11}$ is nonsingular. Moreover, the dimensions $N$ and $M$ may be different from those obtained when applying the variable inversion method. 
Example 3 Consider again the system given in Example 1. Applying the substitutions $z_{1}=\frac{\widetilde{z}_{1}+2}{2 \widetilde{z}_{1}-1}$ and $z_{2}=\frac{\widetilde{z}_{2}+1}{\widetilde{z}_{2}-1}$ to the transfer-function matrix $G_{R P}\left(z_{1}, z_{2}\right)$ gives

$$
\widetilde{G}_{R S}=\left[\begin{array}{cc}
\frac{\widetilde{z}_{1}+2}{3 \widetilde{z}_{1}+1} & 0 \\
-\frac{\left(\widetilde{z}_{1}+2\right)\left(3 \widetilde{z}_{1} \widetilde{z}_{2}+4 \widetilde{z}_{1}+\widetilde{z}_{2}-2\right)\left(\widetilde{z}_{2}-1\right)}{\left(3 \widetilde{z}_{1}+1\right)\left(\left(\widetilde{z}_{2}^{2}-\widetilde{z}_{2}-2\right) \widetilde{z}_{1}-3 \widetilde{z}_{2}^{2}+3 \widetilde{z}_{2}-4\right)} & \frac{\left(\widetilde{z}_{1}+2\right)\left(\widetilde{z}_{2}^{2}-2 \widetilde{z}_{2}+3\right)}{\left(\widetilde{z}_{1}-3\right)\left(\widetilde{z}_{2}^{2}-\widetilde{z}_{2}-2\right)-10}
\end{array}\right] .
$$

Now construct the 3-D polynomial matrix (see (21))

$$
\begin{aligned}
& H_{0}=A_{F}\left(\widehat{z}_{1}, \widetilde{z}_{2}, z\right) \\
& =\left[\begin{array}{c}
z\left(3 \widetilde{z}_{1}+1\right)\left(\widetilde{z}_{1} \widetilde{z}_{2}^{2}-3 \widetilde{z}_{2}^{2}-\widetilde{z}_{1} \widetilde{z}_{2}-2 \widetilde{z}_{1}+3 \widetilde{z}_{2}-4\right)-\left(\widetilde{z}_{1}+2\right)\left(\widetilde{z}_{2} \widetilde{z}_{2}^{2}-3 \widetilde{z}_{2}^{2}-\widetilde{z}_{1} \widetilde{z}_{2}-2 \widetilde{z}_{1}+3 \widetilde{z}_{2}-4\right) \\
\left(\widetilde{z}_{1}+2\right)\left(\widetilde{z}_{2}-1\right)\left(3 \widetilde{z}_{1} \widetilde{z}_{2}+4 \widetilde{z}_{1}+\widetilde{z}_{2}-2\right)
\end{array}\right. \\
& \left.\left.\left.z\left(\widetilde{z}_{1} \widetilde{z}_{2}^{2}-\widetilde{z}_{1} \widetilde{z}_{2}-2 \widetilde{z}_{1}-3 \widetilde{z}_{2}^{2}+3 \widetilde{z}_{2}-4\right)-\left(\widetilde{z}_{2}^{2}-2 \widetilde{z}_{2}+3\right)\left(\widetilde{z}_{1}+2\right)\right]\right)\right]
\end{aligned}
$$

where $z$ is an auxiliary variable.

Next apply, as in the previous example, the following sequence of EOA op- 
erations.

$$
\begin{aligned}
& H_{1}=\operatorname{Augment}\left(H_{0}\right), H_{1}^{1}=\operatorname{addrow}\left(H_{1}, 1,2, z\right), \\
& H_{1}^{2}=\operatorname{addcol}\left(H_{1}^{1}, 1,2,-\left(3 \widetilde{z}_{1}+1\right)\left(\widetilde{z}_{1} \widetilde{z}_{2}^{2}-3 \widetilde{z}_{2}^{2}-\widetilde{z}_{1} \widetilde{z}_{2}-2 \widetilde{z}_{1}+3 \widetilde{z}_{2}-4\right)\right), \\
& H_{2}=\operatorname{Augment}\left(H_{1}^{2}\right), H_{2}^{1}=\operatorname{addrow}\left(H_{2}, 1,4, z\right), \\
& H_{2}^{2}=\operatorname{addcol}\left(H_{2}^{1}, 1,4,-\left(\widetilde{z}_{1} \widetilde{z}_{2}^{2}-3 \widetilde{z}_{2}^{2}-\widetilde{z}_{1} \widetilde{z}_{2}-2 \widetilde{z}_{1}+3 \widetilde{z}_{2}-4\right)\right), \\
& H_{3}=\operatorname{Augment}\left(H_{2}^{2}\right), H_{3}^{1}=\operatorname{addcol}\left(H_{3}, 1,4,\left(\widetilde{z}_{1} \widetilde{z}_{2}^{2}-3 \widetilde{z}_{2}^{2}-\widetilde{z}_{1} \widetilde{z}_{2}-2 \widetilde{z}_{1}+3 \widetilde{z}_{2}-4\right)\right) \text {, } \\
& H_{3}^{2}=\operatorname{addrow}\left(H_{3}^{1}, 1,3,\left(3 \widetilde{z}_{1}+1\right)\right), \quad H_{3}^{3}=\operatorname{addrow}\left(H_{3}^{2}, 1,4,\left(\widetilde{z}_{1}+2\right)\right), \\
& H_{3}^{4}=\operatorname{addrow}\left(H_{3}^{3}, 3,4,-\frac{1}{3}\right), \\
& H_{4}=\operatorname{Augment}\left(H_{3}^{4}\right), \quad H_{4}^{1}=\operatorname{addcol}\left(H_{4}, 1,5,\left(\widetilde{z}_{1}+2\right)\right), \quad H_{4}^{2}=\operatorname{addrow}\left(H_{4}^{1}, 1,2,\left(-\widetilde{z}_{2}^{2}+\widetilde{z}_{2}+2\right)\right) \text {, } \\
& H_{4}^{3}=\operatorname{addrow}\left(H_{4}^{2}, 1,6,-\left(\widetilde{z}_{2}-1\right)\left(3 \widetilde{z}_{1} \widetilde{z}_{2}+\widetilde{z}_{2}+4 \widetilde{z}_{1}-2\right)\right), \\
& H_{5}=\operatorname{Augment}\left(H_{4}^{1}\right), \quad H_{5}^{1}=\operatorname{addcol}\left(H_{5}, 1,2,\left(\widetilde{z}_{2}-1\right)\right), \\
& H_{5}^{2}=\operatorname{addrow}\left(H_{5}^{1}, 1,7,\left(3 \widetilde{z}_{1} \widetilde{z}_{2}+4 \widetilde{z}_{1}+\widetilde{z}_{2}-2\right)\right) \text {, } \\
& H_{5}^{3}=\operatorname{addrow}\left(H_{5}^{2}, 1,3,\left(\widetilde{z}_{2}-1\right)\right), \quad H_{5}^{4}=\operatorname{addrow}\left(H_{5}^{3}, 1,3,1\right) \text {, } \\
& H_{6}=\operatorname{Augment}\left(H_{5}^{4}\right), \quad H_{6}^{1}=\operatorname{addrow}\left(H_{6}, 1,8, \widetilde{z}_{1}\right), \quad H_{6}^{2}=\operatorname{addcol}\left(H_{6}^{1}, 1,2,-3\left(\widetilde{z}_{2}+4\right)\right), \\
& H_{6}^{3}=\operatorname{addcol}\left(H_{6}^{2}, 1,8,\left(\widetilde{z}_{2}^{2}-2 \widetilde{z}_{2}+3\right)\right) \text {, } \\
& H_{7}=\operatorname{Augment}\left(H_{6}^{3}\right), \quad H_{7}^{1}=\operatorname{addrow}\left(H_{7}, 1,6, \widetilde{z}_{1}\right), \quad H_{7}^{2}=\operatorname{addcol}\left(H_{7}^{1}, 1,9,\left(\widetilde{z}_{2}^{2}-\widetilde{z}_{2}-2\right)\right), \\
& H_{8}=\operatorname{Augment}\left(H_{7}^{2}\right), H_{8}^{1}=\operatorname{addcol}\left(H_{8}, 1,10, \widetilde{z}_{2}\right), H_{8}^{2}=\operatorname{addrow}\left(H_{8}^{1}, 1,2,\left(-\widetilde{z}_{2}+1\right)\right) \text {, } \\
& H_{8}^{3}=\operatorname{addrow}\left(H_{8}^{2}, 1,3,\left(-\widetilde{z}_{2}+2\right)\right), \quad H_{8}^{4}=\operatorname{addrow}\left(H_{8}^{3}, 1,7,\left(-3 \widetilde{z}_{2}+3\right)\right) \text {, } \\
& H_{8}^{5}=\operatorname{addrow}\left(H_{8}^{4}, 1,10,\left(2 \widetilde{z}_{2}-4\right)\right), H_{8}^{6}=\operatorname{addrow}\left(H_{8}^{5}, 2,3,-1\right), H_{8}^{7}=\operatorname{addrow}\left(H_{8}^{6}, 2,7,-3\right) \text {, } \\
& H_{8}^{8}=\operatorname{addrow}\left(H_{8}^{7}, 2,10,2\right), H_{8}^{9}=\operatorname{addrow}\left(H_{8}^{8}, 3,6, \frac{1}{3}\right), H_{8}^{10}=\operatorname{addrow}\left(H_{8}^{9}, 3,10, \frac{1}{3}\right), \\
& H_{9}=\operatorname{Augment}\left(H_{8}^{10}\right), \quad H_{9}^{1}=\operatorname{addcol}\left(H_{9}, 1,4, \widetilde{z}_{1}\right), \quad H_{9}^{2}=\operatorname{addrow}\left(H_{9}^{1}, 1,11,-1\right) \text {, } \\
& H_{10}=\operatorname{Augment}\left(H_{9}^{2}\right), \quad H_{10}^{1}=\operatorname{addcol}\left(H_{10}, 1,11, \widetilde{z}_{2}\right), \quad H_{10}^{2}=\operatorname{addrow}\left(H_{10}^{1}, 1,8,5 \widetilde{z}_{2}-5\right), \\
& H_{11}=\operatorname{Augment}\left(H_{10}^{2}\right), \quad H_{11}^{1}=\operatorname{addrow}\left(H_{11}, 1,2, \widetilde{z}_{2}\right), H_{11}^{2}=\operatorname{addcol}\left(H_{11}^{1}, 1,12,-1\right), \\
& H_{11}^{3}=\operatorname{mulrow}\left(H_{11}^{2}, 5,-1\right), H_{11}^{4}=\operatorname{mulrow}\left(H_{11}^{3}, 6,-\frac{1}{3}\right), H_{11}^{5}=\operatorname{mulrow}\left(H_{11}^{4}, 9, \frac{1}{5}\right) \text {, } \\
& H_{11}^{6}=\operatorname{mulrow}\left(H_{11}^{5}, 11, \frac{1}{3}\right) \text {. }
\end{aligned}
$$


Finally, applying appropriate row and column permutations and ordering to the result of this procedure followed by block splitting gives the following matrices of the singular Roesser model (23)

$$
\begin{aligned}
& H_{11}=\left[\begin{array}{ccccc}
0 & 0 & 1 & 0 & 0 \\
-1 & 0 & 0 & 0 & 0 \\
0 & 0 & -2 & 0 & 0 \\
0 & 0 & 0 & 3 & 0 \\
0 & 0 & 0 & 0 & -\frac{1}{3}
\end{array}\right], H_{12}=\left[\begin{array}{cccccc}
-1 & 0 & 0 & 0 & 0 & 0 \\
0 & 0 & 0 & 0 & 0 & 0 \\
0 & 0 & 0 & 0 & -1 & 0 \\
0 & -10 & 0 & 0 & 0 & 0 \\
0 & 0 & 0 & 0 & 0 & 0
\end{array}\right], H_{13}=\left[\begin{array}{cc}
0 & 0 \\
0 & 0 \\
0 & 0 \\
0 & -1 \\
-\frac{1}{3} & 0
\end{array}\right], \\
& H_{21}=\left[\begin{array}{ccccc}
0 & 0 & 0 & 0 & 0 \\
0 & 0 & 0 & 0 & 0 \\
0 & 0 & 0 & 1 & 0 \\
0 & \frac{1}{3} & 0 & -\frac{1}{3} & 0 \\
0 & 0 & 0 & 0 & 0 \\
0 & -\frac{1}{15} & 0 & \frac{1}{15} & -\frac{1}{5}
\end{array}\right], H_{22}=\left[\begin{array}{cccccc}
0 & 0 & 0 & 0 & 0 & -1 \\
0 & 0 & -1 & 0 & 0 & 0 \\
0 & -2 & 1 & 0 & 0 & 0 \\
0 & \frac{5}{3} & \frac{1}{3} & -\frac{4}{3} & 0 & 0 \\
0 & 0 & 0 & -1 & 1 & 0 \\
0 & -\frac{1}{3} & -\frac{1}{15} & \frac{4}{15} & -\frac{2}{5} & 1
\end{array}\right], H_{23}=\left[\begin{array}{cc}
0 & 0 \\
0 & 0 \\
0 & 0 \\
0 & 0 \\
0 & 0 \\
0 & 0
\end{array}\right], \\
& H_{31}=\left[\begin{array}{ccccc}
0 & 0 & 0 & 0 & -\frac{5}{3} \\
1 & -\frac{1}{3} & 0 & -\frac{5}{3} & 0
\end{array}\right], H_{32}=\left[\begin{array}{cccccc}
0 & 0 & 0 & 0 & 0 & 0 \\
0 & \frac{25}{3} & \frac{5}{3} & \frac{10}{3} & 0 & 0
\end{array}\right], H_{33}=\left[\begin{array}{lll}
0 & 0 \\
0 & 0
\end{array}\right],
\end{aligned}
$$

where

$$
E^{\prime}=\left[\begin{array}{cc}
0 & 0 \\
0 & I_{10}
\end{array}\right]
$$

Computing the relevant matrices yields the nonsingular Roesser model with the following matrices

$$
\begin{aligned}
A_{11} & =\left[\begin{array}{ccccc}
\frac{1}{2} & 0 & 0 & 0 & 0 \\
0 & \frac{1}{2} & 0 & \frac{1}{2} & 0 \\
0 & 0 & 0 & 0 & -\frac{3}{10} \\
0 & 0 & 0 & 0 & 0 \\
0 & 0 & 0 & 0 & -1
\end{array}\right], A_{12}=\left[\begin{array}{cccccc}
0 & 0 & 0 & 0 & 0 & 0 \\
0 & \frac{5}{2} & 0 & \frac{15}{2} & -\frac{35}{2} & 0 \\
0 & 0 & 0 & \frac{1}{2} & -\frac{1}{2} & \frac{5}{2} \\
0 & 0 & 5 & 0 & 0 & 0 \\
0 & 0 & 0 & 0 & 0 & 0
\end{array}\right], B_{1}=\left[\begin{array}{cc}
0 & 0 \\
0 & -1 \\
\frac{1}{5} & 0 \\
0 & 1 \\
1 & 0
\end{array}\right], \\
A_{21} & =\left[\begin{array}{ccccc}
-1 & 0 & -\frac{2}{5} & 0 & -\frac{6}{25} \\
0 & 0 & 0 & -\frac{1}{5} & 0 \\
0 & 0 & 0 & \frac{1}{5} & 0 \\
0 & 0 & 0 & 0 & 0 \\
0 & 0 & 0 & 0 & \frac{3}{5} \\
1 & 0 & \frac{2}{5} & 0 & \frac{6}{25}
\end{array}\right], A_{22}=\left[\begin{array}{cccccc}
1 & 0 & 0 & \frac{2}{5} & -\frac{2}{5} & 2 \\
0 & 1 & 1 & 0 & 0 & 0 \\
0 & -2 & 0 & 0 & 0 & 0 \\
0 & 0 & 0 & 1 & -2 & 0 \\
0 & 0 & 0 & -1 & 2 & -5 \\
-2 & 0 & 0 & -\frac{2}{5} & \frac{2}{5} & -1
\end{array}\right], B_{2}=\left[\begin{array}{ccc}
\frac{4}{25} & 0 \\
0 & \frac{2}{5} \\
0 & -\frac{2}{5} \\
0 & 0 \\
-\frac{2}{5} & 0 \\
-\frac{4}{25} & 0
\end{array}\right], \\
C_{1} & =\left[\begin{array}{lllllll}
0 & 0 & 0 & 0 & -1 \\
0 & \frac{1}{2} & 0 & \frac{1}{2} & 0
\end{array}\right], C_{2}=\left[\begin{array}{llllll}
0 & 0 & 0 & 0 & 0 & 0 \\
0 & \frac{5}{2} & 0 & \frac{5}{2} & -\frac{5}{2} & 0
\end{array}\right], \quad D=\left[\begin{array}{cc}
1 & 0 \\
0 & -1
\end{array}\right] .
\end{aligned}
$$

Finally, it is easily verified that the transfer-function matrix corresponding to this nonsingular Roesser state-space model is the same as the initial one, i.e., as given in (17).

\section{Conclusions and Open Research Questions}

In this paper a procedure for obtaining an equivalent 2-D nonsingular Roesser state-space model description for a given system matrix arising from a discrete 
linear repetitive process has been developed. The connection between the original system matrix and its corresponding 2-D nonsingular Roesser state-space model representation has been obtained using elementary operations and the application of i) an auxiliary variables transform and its inversion and ii) a generalized bilinear transform. For a given 2-D transfer-function matrix this new procedure gives a variety of input-output equivalent state-space models. This makes it possible to use different variable transforms and apply different strings of elementary operations corresponding to a particular choice of transform. The $\mathrm{I} / \mathrm{O}$ behavior of the original system matrix is preserved, making it possible to analyze the polynomial system matrix in terms of its associated 2-D nonsingular Roesser state-space model. One motivation for this work is that nonsingular 2-D representations are critical to examining certain physically well defined systems theoretic properties. Moreover, the Roesser model is considered in this paper but the analysis extends directly to the Fornasini Marchesini model.

The results in this paper also show the possibility of obtaining new equivalent forms of repetitive process description, which may have onward value in terms of the development of a comprehensive systems theory that is supported by numerically reliable computational algorithms for checking systems theoretic properties, such as controllability, and the design of control laws. Hence, possible future research includes extending the analysis of this paper to more general forms of repetitive process dynamics. For example, in [17] it is established that cases exist where the pass state initial vector sequence is required to contain explicit terms from the previous pass profile, e.g.,

$$
x_{k+1}(0)=d_{k+1}+\sum_{j=1}^{M} \sum_{p=0}^{\alpha-1} K_{j p} y_{k+1-j}(p), \quad k \geq 0 \text {. }
$$

If the summation term is removed then the state initial vector sequence assumed in this paper results. However, under-modeling, e.g., assuming that this assumption can always be made is incorrect as the structure of the pass state initial vector sequence alone can destroy stability and properties such as controllability/reachability. An example demonstrating this fact is given in [17]. Another application is for the so-called wave repetitive processes characterized by non-local updating structures see e.g. [3].

\section{References}

[1] S. Attasi. Systemes lineaires a deux indices. Technical Report 31, IRIA, France, 1973.

[2] M.S. Boudellioua, K. Galkowski, and E. Rogers. On the connection between discrete linear repetitive processes and 2-D discrete linear systems. Multidimensional Systems and Signal processing, (DOI 10.1007/s11045-016-04548):11 pages, 2016. 
[3] B Cichy, K. Galkowski, E. Rogers, and A. Kummert. Control law design for discrete linear repetitive processes with non-local updating structures. Multidimensional Systems and Signal Processing, 24(4):707-726, 2013.

[4] E. Fornasini and G. Marchesini. State space realization theory of two-dimensional filters. IEEE Transactions on Automatc Control, AC21(4):484-492, 1976.

[5] K. Galkowski. State space realization of linear 2-D systems with extensions to the general $n D(n>2)$ case. Springer, London, 2001.

[6] K. Galkowski, E. Rogers, and D. H. Owens. Matrix rank based tests for reachabiltiy/controllability of discrete linear repetitive processes. Linear Algebra and its Applications, 275-276:201-224, 1998.

[7] K Galkowski, E. Rogers, and D. H. Owens. New 2D models and a transition matrix for discrete linear repetitive processes. International Journal of Control, 72(5):1365-1380, 1999.

[8] L. Hladowski, K. Galkowski, Z. Cai, E. Rogers, C. T. Freeman, and P.L. Lewin. Experimentally supported 2D systems based iterative learning control law design for error convergence and performance. Control Engineering Practice, 18:339- 348, 2010.

[9] Lukasz Hladowski, Krzysztof Galkowski, Zhonglun Cai, Eric Rogers, Chris. T. Freeman, and Paul L. Lewin. Output information based iterative learning control law design with experimental verification. ASME Journal of Dynamic Systems, Measurement and Control, 134(2):021012/1021012/10, 2012.

[10] D.S. Johnson. Coprimeness in multidimensional system theory and symbolic computation. $\mathrm{PhD}$ thesis, Loughborough University of Technology, UK, 1993.

[11] D.S. Johnson, A.C. Pugh, E. Rogers, G.E. Hayton, and D .H. Owens. A polynomial matrix theory for a certain class of two-dimensional linear systems. Linear Algebra and its Applications, 241-243(JulyAugust):669$703,1996$.

[12] B.C. Levy. 2-D polynomial and rational matrices and their applications for the modelling of 2-D dynamical systems. PhD thesis, Stanford University, USA, 1981.

[13] Wojciech Paszke, Eric Rogers, and Krzysztof Galkowski. Experimentally verified generalized kyp lemma based iterative learning control design. Control Engineering Practice, 53:57-67, 2016.

[14] A.C. Pugh, S.J. McInerney, M.S. Boudellioua, D.S. Johnson, and G.E. Hayton. A transformation for 2-D linear systems and a generalization of a theorem of Rosenbrock. International Journal Control, 71(3):491-503, 1998. 
[15] A.C. Pugh, S.J. McInerney, M. Hou, and G.E. Hayton. A transformation for 2-D systems and its invariants. In Proceedings of the 35th IEEE conference on Decision and Control, pages 2157-2158, Kobe (Japan), 1996.

[16] R.P. Roesser. A discrete state-space model for linear image processing. IEEE Transactions on Automatc Control, AC-20(1):1-10, 1975.

[17] E. Rogers, K. Galkowski, and D. H. Owens. Control Systems Theory and Applications for Linear Repetitive Processes. Control and Information Sciences. Springer-Verlag, Berlin Heidelberg, 2007.

[18] H. H. Rosenbrock. State space and multivariable theory. Nelson-Wiley, London, New York, 1970.

[19] Li Xu, Shi Yan, Zhiping Lin, and S. Matsushita. A new elementary operation approach to multidimensional realization and LFR uncertainty modeling: The MIMO case. IEEE Transactions on Circuits and Systems-I, 59(3):638-651, 2012. 